\title{
Expert Cognitive Control and Individual Differences Associated with Frontal and Parietal White Matter Microstructure
}

\author{
R. Edward Roberts, ${ }^{1}$ Elaine J. Anderson, ${ }^{2}$ and Masud Husain ${ }^{2,3}$ \\ ${ }^{1}$ University of Cambridge, Faculty of Economics, Cambridge, CB3 9DD, United Kingdom, ${ }^{2}$ UCL Institute of Cognitive Neuroscience, University College \\ London, London WC1N 3AR, United Kingdom, and ${ }^{3}$ UCL Institute of Neurology, University College London, London WC1N 3BG, United Kingdom
}

\begin{abstract}
Although many functional imaging studies have reported frontal activity associated with "cognitive control" tasks, little is understood about factors underlying individual differences in performance. Here we compared the behavior and brain structure of healthy controls with fighter pilots, an expert group trained to make precision choices at speed in the presence of conflicting cues. Two different behavioral paradigms - Eriksen Flanker and change of plan tasks - were used to assess the influence of distractors and the ability to update ongoing action plans. Fighter pilots demonstrated superior cognitive control as indexed by accuracy and postconflict adaptation on the Flanker task, but also showed increased sensitivity to irrelevant, distracting choices. By contrast, when pilots were examined on their ability to inhibit a current action plan in favor of an alternative response, their performance was no better than the control group.

Diffusion weighted imaging revealed differences in white matter radial diffusivity between pilots and controls not only in the right dorsomedial frontal region but also in the right parietal lobe. Moreover, analysis of individual differences in reaction time costs for conflict trials on the Flanker task demonstrated significant correlations with radial diffusivity at these locations, but in different directions. Postconflict adaptation effects, however, were confined to the dorsomedial frontal locus. The findings demonstrate that in humans expert cognitive control may surprisingly be mediated by enhanced response gain to both relevant and irrelevant stimuli, and is accompanied by structural alterations in the white matter of the frontal and parietal lobe.
\end{abstract}

\section{Introduction}

What makes someone an expert? In elite athletes, much of the focus has been on physiological and metabolic attributes in cardiovascular, respiratory and skeletomuscular systems. However, it has become apparent even in sports that expert performance relies also on cognitive processes (Yarrow et al., 2009). Although previous studies have reported increases in cortical volume associated with expertise in a number of fields (Draganski and May, 2008), the relationship between white matter structure and expertise is still poorly understood. Investigations into the neural basis of exceptional musical performance have reported both increases and decreases in measures of white matter microstructure relative to novices (Imfeld et al., 2009).

Recently there has been interest in whether there might also be white matter changes associated with superior cognitive control on tasks in which participants have to select between conflicting responses. Some studies using such tasks have shown alterations in white matter with development, or between patient and healthy controls (Liston et al., 2006). However, to date there has

Received Sept. 17, 2010; revised 0ct. 14, 2010; accepted 0ct. 20, 2010.

This research was supported by the Wellcome Trust, the Medical Research Council, and the National Institute for Health Research Clinical Biomedical Centre at University College London Hospitals/University College London. Many thanks to Dr. Olga Ciccarelli for helpful technical discussions on this manuscript, to Dr. Parashkev Nachev for advice on the use of the change of plan task, and to the RAF pilots and volunteers who took part in this study.

Correspondence should be addressed to R. Edward Roberts at the above address. E-mail: rer40@cam.ac.uk.

DOI:10.1523/JNEUROSCI.4879-10.2010

Copyright $\odot 2010$ the authors $\quad$ 0270-6474/10/3017063-05\$15.00/0 been no report of a professional group which demonstrates superior cognitive control associated with white matter microstructural markers.

To examine this issue we assessed a group of individuals who are trained to deal with exceptionally demanding situations, where elevated levels of cognitive control are crucial. Royal Air Force (RAF) fighter pilots are highly trained in specialist low altitude flight at supersonic speeds, a discipline that is considered to be at the limit of human cognitive capability as it requires fine control with very little room for error (Haber and Haber, 2003). We compared the performance of a group of Tornado fighter pilots with a group of control subjects on two measures of cognitive control and then used diffusion tensor imaging (DTI) to measure the passive diffusion of water molecules in the brain-a technique used to infer properties of the underlying white matter microstructure (Beaulieu, 2002).

An influential view of how the brain achieves control in situations where there are conflicting choices proposes involvement of brain regions located in medial and lateral frontal cortex (MacDonald et al., 2000). Functional brain imaging studies have consistently demonstrated increased activity in frontal areas during the performance of so-called "response conflict tasks" (MacDonald et al., 2000; Ullsperger and von Cramon, 2001; Ridderinkhof et al., 2004; Rushworth et al., 2004), but recent research suggests that posterior parietal brain regions might be equally important (Coulthard et al., 2008). We investigated whether elite pilots demonstrate superior cognitive control and if behavioral 

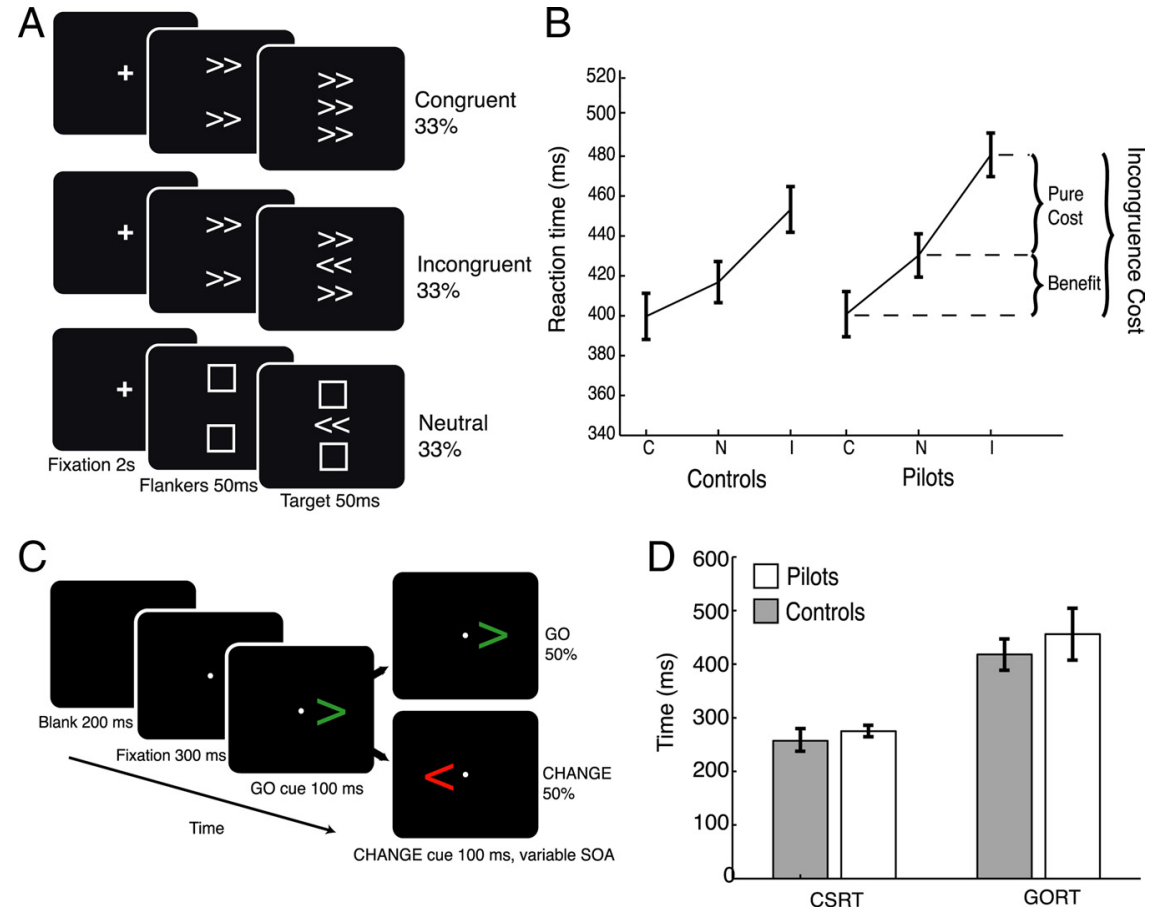

Figure 1. Experimental procedure and behavioral results. $A$, In the Eriksen Flanker task subjects respond to the direction of the central arrow. This is flanked by arrows in the same direction (congruent), the opposite direction (incongruent), or squares (neutral). $\boldsymbol{B}$, The pilots demonstrated significantly increased incongruence costs (pure cost, benefit and incongruence cost), but did not show significant differences in RT at the group level. $C$, Congruent; $\mathrm{N}$, neutral; I, incongruent stimuli. $C$, In the change of plan task subjects respond to the green $\mathrm{GO}$ signal, unless they see a red CHANGE arrow. $\boldsymbol{D}$, There was no significant different between pilots and controls on this task. Error bars indicate \pm SEM.

advantages are accompanied by differences in the white matter adjacent to the frontal and parietal regions known to be involved in such control.

\section{Materials and Methods}

Eleven male RAF Tornado pilots and an age- and sex-matched control group $(n=11)$ with no previous experience of piloting aircraft gave written informed consent to participate in this study, which was approved by a local ethics committee. The experts and the controls were all right-handed and matched for IQ (Table S1, available at www.jneurosci. org as supplemental material).

All stimuli were displayed using Presentation software (Neurobehavioral Systems). In the Eriksen Flanker task, participants were presented with three equi-probable trial types: congruent, incongruent, and neutral (Fig. 1 A) (Eriksen and Eriksen, 1974). A fixation cross was presented for $2 \mathrm{~s}$ followed by flankers for $50 \mathrm{~ms}$, and then the target plus flankers for a further $50 \mathrm{~ms}$. Observers were instructed to respond as quickly and accurately as possible to the direction of a central target arrow (left or right) by pressing the corresponding button - with left or right index fingerswhile ignoring the peripheral flanking arrows. They were required to perform six blocks of 60 trials with short rest periods between.

In the change of plan task (Nachev et al., 2005) participants were presented with a single green arrow (GO signal) on $50 \%$ of trials. On the remaining trials the Go signal was followed by a red arrow pointing in the opposite direction (CHANGE signal). Subjects were instructed to respond as quickly as possible to the GO signal unless they saw a CHANGE signal, in which case they should respond instead to the direction of the CHANGE cue. This paradigm is analogous to the STOP task, widely used to measure response inhibition (Aron and Poldrack, 2006).

The delay between GO and CHANGE signals_-change signal delay (CSD) - was varied parametrically in response to the performance of the participant using an adaptive staircase technique. The change-signal reaction time (CSRT), which reflects the time taken to inhibit an initial response and successfully execute an alternate response on $50 \%$ of change trials, was calculated in analogous manner to that used in the STOP paradigm (Logan, 1994). A cubic spline was fitted to the changesignal reaction time data, guided by the distribution of Go responses, using the local variation of Go responses to interpolate between change trial data points. The CSD was then subtracted from the spline values at each point, the median of which gave the value of CSRT. A shorter CSRT implies that response plans can be changed at a later point in the process, indicating greater cognitive flexibility. Participants performed 8 blocks of 40 experimental trials, with short rest periods between.

MRI data acquisition. MRI data were acquired using a 1.5T Siemens Magnetom Vision system. Four sets of whole brain diffusion weighted volumes were acquired [12 directions; $b=1000 \mathrm{~s}$ $\mathrm{mm}^{-2}$; 48 slices; voxel size $2 \times 2 \times 3 \mathrm{~mm}^{3}$; repetition time $(\mathrm{TR})=8.6 \mathrm{~s}$; echo time $(\mathrm{TE})=94$ $\mathrm{ms})$, plus four volumes without diffusion weighting $\left(b=0 \mathrm{~s} \mathrm{~mm}^{-2}\right)$. A T1-weighted anatomical image was acquired using a magnetizationprepared rapid-acquisition gradient echo sequence $(\mathrm{TR}=1160 \mathrm{~ms}$; $\mathrm{TE}=4.38 \mathrm{~ms}$; flip angle $=15$; voxel size $1 \times 1 \times 1 \mathrm{~mm}^{3}$ ).

Voxelwise statistical analysis of the diffusion-weighted data was performed using tract-based spatial statistics, part of FSL (Smith et al., 2004). Fractional anisotropy (FA) images were created by fitting a tensor model to the raw diffusion data using FDT, and then brainextracted using BET. All subjects' FA data were then aligned into a common space using the nonlinear registration tool FNIRT. A mean FA image was created and thinned to generate a mean FA skeleton that represents the centers of all tracts common to the group. Each subject's aligned FA data were then projected onto this skeleton. Longitudinal $\left(D_{L}\right)$ and radial direction $\left(D_{R}\right)$ maps were also generated from the DTI.

A region of interest (ROI) approach was used to test for differences in the white matter diffusivity between the two groups. ROIs were chosen based on the contrasts from a functional imaging study using a change of plan task (Nachev et al., 2005), and closely matched the medial frontal and parietal activations reported from a study using a Flanker task (Ullsperger and von Cramon, 2001) (see supplemental Fig. S1, supplemental Table S2, available at www.jneurosci.org as supplemental material). Four brain areas were selected, represented bilaterally and comprised; dorsomedial frontal cortex (DMFC), parietal cortex (PC), inferior frontal cortex (IFC), and dorsolateral prefrontal cortex (DLPFC). White matter ROIs (mean $=267$ voxels, $S D=53.6$ ) were generated using the overlap between the fMRI activations and the white matter skeleton, with three slices taken anterior and posterior to the centroid of activation (supplemental Fig. S1, available at www.jneurosci.org as supplemental material).

A voxelwise comparison analysis between the groups of $D_{L}$ and $D_{R}$ in each ROI was performed with clusters thresholded at $t>2$ and tested for significance at $p<0.05$, familywise error (FWE) corrected for multiple comparisons using permutation-based nonparametric testing (Nichols and Holmes, 2002), for clusters of $>10$ contiguous voxels. The mean $D_{R}$ was calculated for significant clusters to investigate the relationship with performance on the Flanker task.

\section{Results}

\section{Behavior on Eriksen Flanker task}

The pilots were significantly more accurate than the control group (pilots $=97.5 \%$, controls $=93.1 \% ; t=2.4, p<0.02$, $\mathrm{df}=20$ ), demonstrating superior control ability. Importantly, this effect could not be explained by slower responses (i.e., a speed-accuracy trade-off) as there was no significant differ- 
ence between the groups' mean reaction times in any of the conditions $(t=1.7, p>0.1, \mathrm{df}=20)$. Thus, pilots were able to perform the task at the same speed as controls but with significantly higher accuracy.

Performance on this paradigm is also measured by comparing the relative differences in reaction time (RT) between conditions, providing three indices of performance (Fig. $1 \mathrm{~B}$ ): pure cost (incongruent-neutral condition), benefit (neutral-congruent), and incongruence cost (incongruent-congruent). These measures are often used to estimate the level of positive (facilitating) and negative (interference) effects on RT evoked by flankers, with higher incongruence costs usually regarded as indicative of poorer cognitive control.

Importantly, although the pilots were more accurate, they also demonstrated a significantly larger pure cost than controls $(t=$ $2.3, p<0.03, \mathrm{df}=20)$. However, they also had a significantly greater benefit $(t=2.9, p<0.008, \mathrm{df}=20)$. Thus they had a greater incongruence cost compared with controls $(t=3.4, p<$ $0.003, \mathrm{df}=20$ ). These results are remarkable as they reveal that pilots had significantly larger interference (RT) costs than controls (Fig. $1 B$ ), while simultaneously being more accurate. Thus, unlike previous demonstrations of differences in cognitive control between say controls and a patient group (Wylie et al., 2009), superior performance here was associated with increased sensitivity to both facilitating (congruent flankers) as well as distracting, interfering stimuli (incongruent flankers).

Could this be due to attentional effects? If the pilots had more focused selective attention, one would predict that the flanking stimuli would have less effect on their RTs, whereas in fact the pilots were significantly more sensitive to the presence of flankers than the controls. If the pilots had superior sustained attention, one might predict that they would demonstrate a lower intraindividual coefficient of variability (variability in responses to neutral stimuli divided by the mean RT) than the controls (Castellanos et al., 2005). However, there was no significant difference between the groups on this measure $(t=$ 0.38, $p>0.39$, df $=20$ ).

Another way to measure performance on cognitive control tasks is to examine how individuals adapt behavior in response to whether a previous trial had been a conflict trial or not-the Gratton effect (Gratton et al., 1992). RT interference effects for postcongruent vs postincongruent (conflict) trials were calculated: (RT on incongruent trial following incongruent trial-RT on incongruent trial following congruent trial). Thus negative values index improved "control" following conflict. Significant within-subject changes in benefit were found in controls $(t=$ $4.79, p<0.001, \mathrm{df}=10)$, and in the pilots' pure cost $(t=2.49$, $p<0.032, \mathrm{df}=10)$ and incongruence cost $(t=4.89, p<0.001$, $\mathrm{df}=10)$ using this method. A between-group analysis revealed that the groups differed significantly only with pure cost following a conflict trial $(t=2.4, p<0.028, \mathrm{df}=20)$. Thus this analysis reveals that pilots demonstrated a significantly better postconflict adaptation effect.

We also performed analyses for two other measures of control: posterror slowing and posterror changes in interference (Rabbitt, 1969; Ridderinkhof, 2002). No significant effect was found within-subjects for posterror slowing in either the pilot $(t=2.0, p>0.07, \mathrm{df}=10$; paired-samples $t$ test) or control group $(t=1.30, p>0.22, \mathrm{df}=9)$; nor were there any significant posterror changes in interference (pilots: $t=1.38, p>0.19$, df $=9$; controls: $t=0.36, p>0.71, \mathrm{df}=9)$. But it has to be borne in mind that error rates were low on this task.

\section{Behavior on change of plan task}

In contrast to the Flanker task, there was no significant difference between the pilots and controls on the change of plan task, either in terms of CSRT $(t=0.58, p>0.40$, df $=20$; independent samples $t$ test), or mean RT to the GO signal $(t=0.95, p>0.51$, $\mathrm{df}=20$ ). Thus on this measure of cognitive control, pilots did not demonstrate superior performance. This finding suggests that expertise in cognitive control may be highly specialized, particular to specific tasks and not simply associated with globally enhanced performance.

\section{White matter analysis}

The diffusion profile within each voxel is comprised of the primary, longitudinal direction of diffusion $\left(D_{L}\right.$, which is usually parallel to the axonal fibers) and the orthogonal secondary and tertiary components, which can be averaged to give the radial diffusivity $\left(D_{R}\right)$. Although a number of studies have combined the three principal diffusivity measurements into a single valueFA-we chose to investigate the principal and radial components of diffusivity separately. There is evidence to suggest that radial diffusivity may be related to the degree of myelination, whereas changes in axial diffusivity could reflect axonal density (Song et al., 2002, 2005). Therefore, analyzing radial and axial components separately may provide more information about the structural basis for any differences observed.

A voxelwise comparison analysis was then performed within our predefined ROIs between the two groups-pilots and controls - using our two measures of diffusivity, $D_{R}$ and $D_{L}$. We found that $D_{R}$ was significantly different in the pilot group than the controls in two right hemisphere regions: in the parietal and frontal lobes (Fig. $2 A, B$ ). In the right parietal cluster adjacent to the intraparietal sulcus (IPS), $D_{R}$ was significantly lower in the pilot group $\left(p<0.05\right.$, corrected, $t_{\max }=3.31, \mathrm{df}=20,[x=41$, $y=-50, z=39])\left(\right.$ pilots $=1.21 \times 10^{-4} \mathrm{~mm}^{2} \mathrm{~s}^{-1}$, controls $=$ $1.41 \times 10^{-4} \mathrm{~mm}^{2} \mathrm{~s}^{-1}$ ) (Fig. $2 \mathrm{~A}$ ). By contrast, in the right dorsomedial frontal (DMF) ROI, $D_{R}$ was significantly higher in the pilot group $\left(p<0.05\right.$, corrected, $t_{\max }=3.6, \mathrm{df}=20,[x=18, y=$ $21, z=41])$ in a cluster of voxels adjacent to dorsomedial premotor areas (pilots $=2.64 \times 10^{-4} \mathrm{~mm}^{2} \mathrm{~s}^{-1}$, controls $=2.22 \times$ $10^{-4} \mathrm{~mm}^{2} \mathrm{~s}^{-1}$ ) (Fig. $2 \mathrm{~B}$ ). No significant differences in radial diffusivity were present between the groups in the inferior frontal or dorsolateral prefrontal white matter regions in either hemisphere, or with respect to the $\mathrm{D}_{\mathrm{L}}$ index in any of the ROIs.

We then examined whether the mean radial diffusivity in the right parietal and frontal clusters correlated with behavioral performance on the Eriksen Flanker task, across individuals. Four measures of performance on the Flanker task that distinguished pilots from controls were used: accuracy, pure cost, benefit, incongruence cost and postconflict pure cost.

Across all participants, mean radial diffusivity in the parietal cluster was significantly negatively correlated with accuracy $(r=$ $-0.64, p<0.001$; two-tailed Pearson correlation). $\mathrm{D}_{\mathrm{R}}$ was also negatively correlated with incongruence cost $(r=-0.52, p<$ 0.02 ) and pure cost $(r=-0.47, p<0.03$ ) (Fig. $2 E-G)$, but not with benefit. By contrast, in the dorsomedial frontal cluster, radial diffusivity was positively correlated with incongruence cost $(r=0.50, p<0.02)$ and benefit $(r=0.48, p<0.02)$, but not with pure cost or accuracy (Fig. $2 C, D$ ). The relationship between mean radial diffusivity and postconflict adaptation effects was also examined. This revealed a significant negative correlation between changes in postconflict pure cost $(r=-0.46, p<0.031)$ and mean diffusivity in the DMFC cluster, but not in the parietal cluster (Fig. $2 H$ ). 

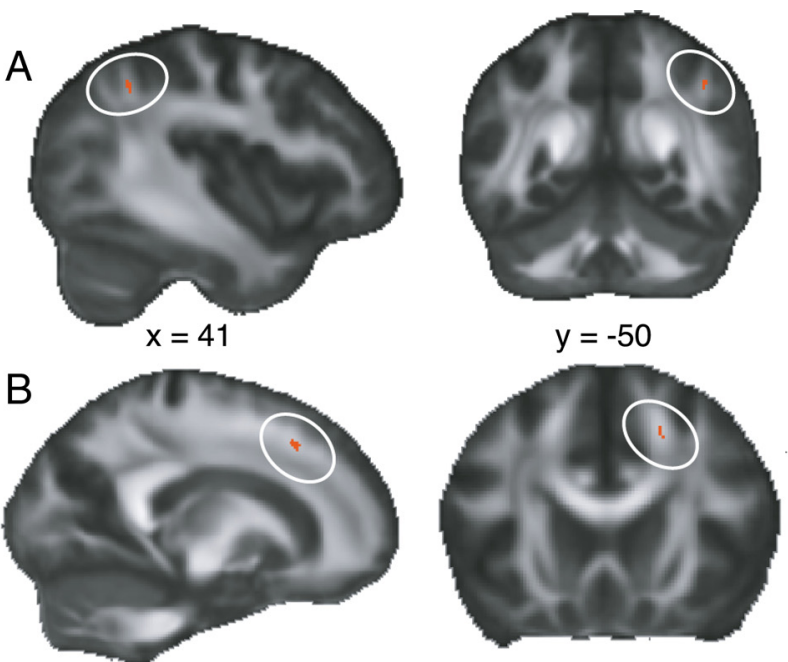

$y=21$

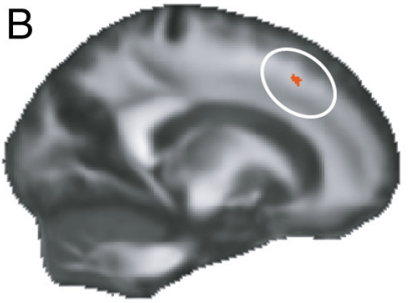

$x=18$
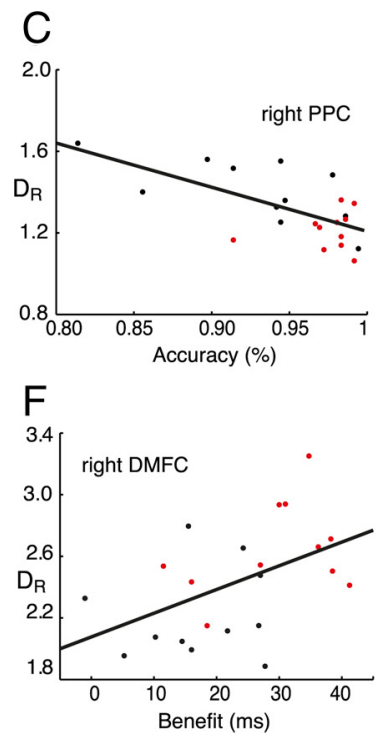

D

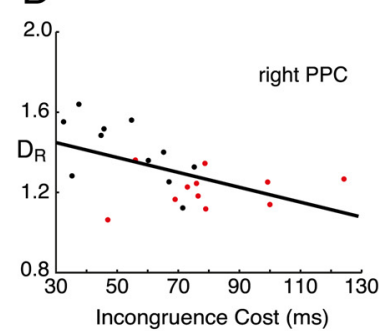

G

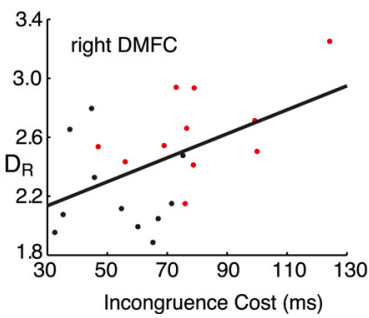

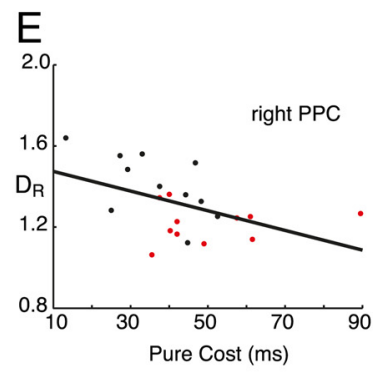

$\mathrm{H}$

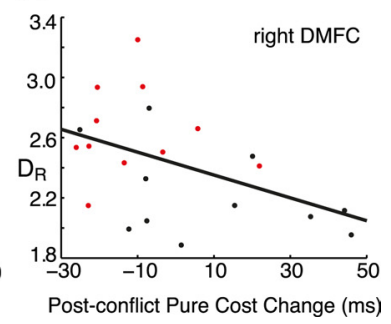

Figure 2. Significant clusters in radial diffusivity contrast analysis. On a mean FA map (grayscale), the locations of differences in radial diffusivity $\left(10^{-4} \mathrm{~mm}^{2} \mathrm{~s}^{-1}\right)$ between pilots and controls are shown in red, circled in white. $A$, In the parietal ROI, we found higher values of radial diffusivity in the control group. $B$, In the DMF ROI, higher values of radial diffusivity occurred in the pilot group. $\mathbf{C}-\boldsymbol{E}$, In the parietal ROI, the mean radial diffusivity was negatively correlated with accuracy, incongruence cost, and pure cost across the group. $\boldsymbol{F}-\boldsymbol{H}$, In the DMFC ROI, the mean radial diffusivity was positively correlated with incongruence cost, benefit, and negatively with postconflict adaptation change in pure cost. Pilots (red), Controls (black).

The results of previous diffusion tensor tractography studies in humans suggest that the parietal cluster lies on the superior longitudinal fasciculus (SLF) (Catani et al., 2002). There is also evidence to suggest that a dorsal subcomponent of SLF may provide a direct connection between posterior parietal cortex-including intraparietal sulcus - to caudal, lateral prefrontal and dorsal premotor regions (Makris et al., 2005; Thiebaut de Schotten et al., 2005; Schmahmann et al., 2007). Therefore it is possible that the differences in radial diffusivity between pilots and controls, and the regions in which there were significant correlations across individuals with performance on the Flanker task, occupy locations in the white matter that connect parietal and frontal cortex in the right hemisphere. However, the existence of this specific component of the SLF in humans is still a matter of debate; further investigation would be required to confirm this hypothesis.

To investigate whether the differences observed might be associated with flying experience, the number of years of general
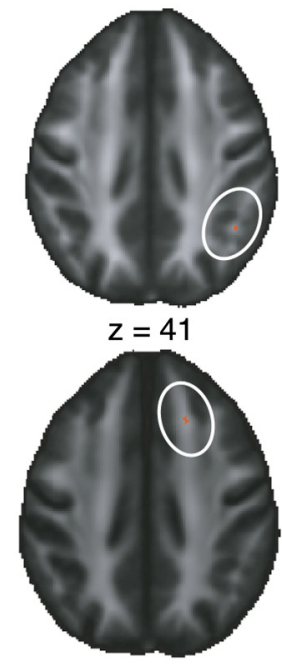

$z=39$

flight training, and the time spent in fast jets was also examined within the pilot group. We found no significant correlations between mean radial diffusivity in the parietal or dorsomedial frontal clusters and general flight experience $(p>$ $0.30)$ or fast jet experience $(p>0.33)$.

\section{Discussion}

The pilots assessed in this study were more accurate than control participants on the Eriksen Flanker task, a widely used measure of cognitive control (Ullsperger and von Cramon, 2001). Importantly, they demonstrated increased sensitivity to both congruent and the distracting, incongruent cues, as indexed by RT effects (Fig. 1B), as well as demonstrating significantly better adaptation following conflict trials (Gratton effect). However, on the change of plan task, which uses the ability to inhibit an initial plan in favor of an alternative response as a measure of control, no significant differences were observed between the groups. Analysis of regions previously associated with brain imaging activations when people perform these tasks revealed differences in radial diffusivity between pilots and controls in two white matter areas of the right hemisphere: one in the frontal lobe and the other in posterior parietal cortex, adjacent to the IPS.

In pilots, there was significantly lower radial diffusivity in the cluster adjacent to right IPS, but higher radial diffusivity in white matter voxels adjacent to the right dorsomedial frontal cortex. Across individuals, $D_{R}$ in both these regions was correlated with sensitivity to the flankers, as indexed by RT costs, but in opposing ways (Fig. $2 C-G$ ), whereas performance accuracy was only correlated with diffusivity in the parietal white matter. Conversely, there was a significant correlation between postconflict RT adaptation and mean diffusivity in the frontal cluster, but not in the parietal one (Fig. $2 H$ ), consistent with the proposal that parietal and DMFC regions may play different roles on processing flanker stimuli (Coulthard et al., 2008). The results of previous studies suggest that the clusters identified here may both lie on a dorsal subcomponent of the SLF pathway connecting superior parietal to frontal regions (Makris et al., 2005; Thiebaut de Schotten et al., 2005; Schmahmann et al., 2007). However, further investigations will be required to establish this, and whether findings from the ROI analysis approach used here can be applied more widely.

It is possible that the lower $\mathrm{D}_{\mathrm{R}}$ observed in the parietal ROI in the pilot group, and higher $D_{R}$ in the frontal region may reflect changes to a network that has been biased to perform optimally in conditions presented by the Flanker task. Functional imaging studies of motor learning suggest that patterns of activity across brain networks may change after learning (Sakai et al., 1998; Toni et al., 2001; Grol et al., 2006). Therefore, long-term learning 
could theoretically result in systematic shifts in brain activity accompanied by changes to white matter structure, with connections to specific brain regions altered to optimize their contribution to network performance.

The physiological basis for the different patterns of correlation-positive and negative-in the two regions we have identified (Fig. 2A,B) cannot currently be established with certainty. A number of factors such as packing density and properties of the cell membrane may all contribute to the diffusion measurement (Beaulieu, 2002). Nonetheless, the crucial result here is that radial diffusivity in human parietal and frontal white matter indexes differences in cognitive control across individuals.

Our findings show that differences in the connections between parietal and frontal areas modulate performance under situations of response conflict. They also demonstrate that increased sensitivity to both incongruent and congruent flankers can be associated with superior accuracy. One potential mechanism might be that increased gain leads to a more robust signal to trigger brain control responses when there are incongruent flanker stimuli. Indeed, a previous functional imaging study suggested that conflict may be resolved through cortical amplification of task-relevant information (Egner and Hirsch, 2005).

It is possible that the changes we have observed between fighter pilots and controls might have developed with training, although no significant correlation was found with duration of expertise. However, it is also conceivable that genetic factors contribute to differences in brain connectivity, particularly since RAF pilots already represent a highly selected group before they begin formal training. Thus, individuals born with such white matter differences might be more suitable for the demands of being a fighter pilot. Such a mechanism raises the possibility that expertise in different cognitive domains-and indeed professions - might be linked to differences in brain connectivity, and not just cortical differences.

\section{References}

Aron AR, Poldrack RA (2006) Cortical and subcortical contributions to Stop signal response inhibition: role of the subthalamic nucleus. J Neurosci 26:2424-2433.

Beaulieu C (2002) The basis of anisotropic water diffusion in the nervous system - a technical review. NMR Biomed 15:435-455.

Castellanos FX, Sonuga-Barke EJ, Scheres A, Di Martino A, Hyde C, Walters JR (2005) Varieties of attention-deficit/hyperactivity disorder-related intraindividual variability. Biol Psychiatry 57:1416-1423.

Catani M, Howard RJ, Pajevic S, Jones DK (2002) Virtual in vivo interactive dissection of white matter fasciculi in the human brain. Neuroimage 17:77-94.

Coulthard EJ, Nachev P, Husain M (2008) Control over conflict during movement preparation: role of posterior parietal cortex. Neuron 58:144-157.

Draganski B, May A (2008) Training-induced structural changes in the adult human brain. Behav Brain Res 192:137-142.

Egner T, Hirsch J (2005) Cognitive control mechanisms resolve conflict through cortical amplification of task-relevant information. Nat Neurosci 8:1784-1790.

Eriksen BA, Eriksen CW (1974) Effects of noise letters upon identification of a target letter in a nonsearch task. Percept Psychophys 16:143-149.

Gratton G, Coles MG, Donchin E (1992) Optimizing the use of information: strategic control of activation of responses. J Exp Psychol Gen 121:480-506.

Grol MJ, de Lange FP, Verstraten FA, Passingham RE, Toni I (2006) Cere- bral changes during performance of overlearned arbitrary visuomotor associations. J Neurosci 26:117-125.

Haber RN, Haber L (2003) Perception and attention during low-altitude high-speed flight. In: Principles and practice of aviation psychology (Tsang PS, Vidulich MA, eds), pp 21-68. Mahwah, NJ: Erlbaum.

Imfeld A, Oechslin MS, Meyer M, Loenneker T, Jancke L (2009) White matter plasticity in the corticospinal tract of musicians: a diffusion tensor imaging study. Neuroimage 46:600-607.

Liston C, Watts R, Tottenham N, Davidson MC, Niogi S, Ulug AM, Casey BJ (2006) Frontostriatal microstructure modulates efficient recruitment of cognitive control. Cereb Cortex 16:553-560.

Logan GD (1994) On the ability to inhibit thought and action: a users' guide to the stop signal paradigm. In: Inhibitory processes in attention, memory, and language (Dagenbach D, Carr TH, eds), pp 189-240. San Diego: Academic.

MacDonald AW 3rd, Cohen JD, Stenger VA, Carter CS (2000) Dissociating the role of the dorsolateral prefrontal and anterior cingulate cortex in cognitive control. Science 288:1835-1838.

Makris N, Kennedy DN, McInerney S, Sorensen AG, Wang R, Caviness VS Jr, Pandya DN (2005) Segmentation of subcomponents within the superior longitudinal fascicle in humans: a quantitative, in vivo, DT-MRI study. Cereb Cortex 15:854-869.

Nachev P, Rees G, Parton A, Kennard C, Husain M (2005) Volition and conflict in human medial frontal cortex. Curr Biol 15:122-128.

Nichols TE, Holmes AP (2002) Nonparametric permutation tests for functional neuroimaging: a primer with examples. Hum Brain Mapp 15:1-25.

Rabbitt P (1969) Psychological refractory delay and response-stimulus interval duration in serial, choice-response tasks. Acta Psychol 30:195-219.

Ridderinkhof KR (2002) Micro- and macro-adjustments of task set: activation and suppression in conflict tasks. Psychol Res 66:312-323.

Ridderinkhof KR, Ullsperger M, Crone EA, Nieuwenhuis S (2004) The role of the medial frontal cortex in cognitive control. Science 306:443-447.

Rushworth MF, Walton ME, Kennerley SW, Bannerman DM (2004) Action sets and decisions in the medial frontal cortex. Trends Cogn Sci $8: 410-417$

Sakai K, Hikosaka O, Miyauchi S, Takino R, Sasaki Y, Pütz B (1998) Transition of brain activation from frontal to parietal areas in visuomotor sequence learning. J Neurosci 18:1827-1840.

Schmahmann JD, Pandya DN, Wang R, Dai G, D'Arceuil HE, de Crespigny AJ, Wedeen VJ (2007) Association fibre pathways of the brain: parallel observations from diffusion spectrum. imaging and autoradiography. Brain 130:630-653.

Smith SM, Jenkinson M, Woolrich MW, Beckmann CF, Behrens TEJ, Johansen-Berg H, Bannister PR, De Luca M, Drobnjak I, Flitney DE (2004) Advances in functional and structural MR image analysis and implementation as FSL. Neuroimage 23:208-219.

Song SK, Sun SW, Ramsbottom MJ, Chang C, Russell J, Cross AH (2002) Dysmyelination revealed through MRI as increased radial (but unchanged axial) diffusion of water. Neuroimage 17:1429-1436.

Song SK, Yoshino J, Le TQ, Lin SJ, Sun SW, Cross AH, Armstrong RC (2005) Demyelination increases radial diffusivity in corpus callosum of mouse brain. Neuroimage 26:132-140.

Thiebaut de Schotten M, Urbanski M, Duffau H, Volle E, Lévy R, Dubois B, Bartolomeo P (2005) Direct evidence for a parietal-frontal pathway subserving spatial awareness in humans. Science 309:2226-2228.

Toni I, Ramnani N, Josephs O, Ashburner J, Passingham RE (2001) Learning arbitrary visuomotor associations: temporal dynamic of brain activity. Neuroimage 14:1048-1057.

Ullsperger M, von Cramon DY (2001) Subprocesses of performance monitoring: A dissociation of error processing and response competition revealed by event-related fMRI and ERPs. Neuroimage 14:1387-1401.

Wylie SA, van den Wildenberg WP, Ridderinkhof KR, Bashore TR, Powell VD, Manning CA, Wooten GF (2009) The effect of Parkinson's disease on interference control during action selection. Neuropsychologia 47:145-157.

Yarrow K, Brown P, Krakauer JW (2009) Inside the brain of an elite athlete: the neural processes that support high achievement in sports. Nat Rev Neurosci 10:585-596. 